\title{
Presentación: Educación científica. Nuevas metodologías para una sociedad cambiante
}

\author{
Apresentação: Educação Científica. Novas metodologias para uma \\ sociedade em constante mudança
}

\author{
F. Javier Perales ${ }^{1}$ \\ David Aguilera ${ }^{2}$ \\ ${ }^{1}$ Universidad de Granada (UGR), España; ${ }^{2}$ Universidad Isabel I (UI1), España
}

En el pasado mes de febrero, desde la Revista Iberoamericana de Educación se convocó un monográfico bajo el título de Educación Científica. Nuevas metodologías para una sociedad cambiante. A lo largo de los meses siguientes y hasta el cierre de aquél se ha venido recibiendo un volumen destacado de propuestas (un total de 54). En una primera fase se efectuó, por parte de los editores, un cribado de las mismas atendiendo a criterios como la alineación de la temática con los objetivos del monográfico descritos en la convocatoria aludida o la naturaleza y calidad de las mismas. Los artículos restantes fueron sometidos a un proceso de evaluación ciega por pares, como es la práctica habitual entre las revistas científicas. Todo ello con la perspectiva temporal de acortar lo máximo posible el intervalo entre la aprobación definitiva de las propuestas y la publicación efectiva, algo que seguramente agradecerán los autores y lectores de este monográfico. El resultado ha sido un número de artículos final que ha propiciado el que hayamos tenido que distribuirlos en dos monográficos consecutivos.

Una sociedad tan cambiante como la nuestra necesita de cambios (innovaciones) y adaptaciones en el ámbito educativo que permitan compaginar lo académico con las demandas sociales. Este primer monográfico estimamos que resulta representativo de la riqueza y diversidad de las propuestas recibidas que, a su vez, constituyen un muestrario de la pujanza de la comunidad de docentes e investigadores en su afán de
No último mês de fevereiro, a Revista Ibero-americana de Educação lançou uma convocatória para o número intitulado Educação Científica. Novas metodologias para uma sociedade em transformação. Durante os meses seguintes e até a data de encerramento, muitas propostas foram recebidas (um total de 54). Na primeira fase, os editores realizaram uma triagem de acordo com determinados critérios, como o alinhamento do tema com os objetivos da publicação descritos na chamada e a natureza e qualidade dos artigos. Os demais artigos foram submetidos a um processo de revisão cega por pares, como é prática comum entre as revistas científicas. Tudo isso foi feito visando a reduzir ao máximo o intervalo entre a aprovação definitiva das propostas e a sua publicação efetiva, algo pelo qual os autores e leitores certamente agradecerão. O resultado foi um número final de artigos, que nos levou a distribuí-los em dois volumes consecutivos.

Uma sociedade em constante transformação como a nossa precisa de mudanças (inovações) e adaptações no campo da educação que permitam combinar o mundo acadêmico com as demandas sociais. Acreditamos que este primeiro volume representa a riqueza e diversidade das propostas recebidas que, por sua vez, constituem uma amostra da força da comunidade de professores e pesquisadores no seu desejo de fazer do ensino de Ciências uma oferta atraente e eficiente para as novas gerações.

Entre os artigos que apresentamos, há estudos focados na formação de docentes. Por um lado, os professores Radu Bogdan 
hacer de la enseñanza de las ciencias una oferta atractiva y eficiente para las nuevas generaciones.

Entre los artículos que presentamos encontrarán estudios centrados en la formación del profesorado. Por un lado, los profesores Radu Bogdan (Universidad de Burgos, España) y Diego Armando Retana (Universidad de Costa Rica, Costa Rica) atienden al conocimiento didáctico de futuros maestros. Por el otro, el profesor Antonio García-Carmona (Universidad de Sevilla, España) y la profesora María José Vargas-Straube junto a sus colaboradores (Universidad Alberto Hurtado, Chile) se ocupan del conocimiento científico-tecnológico de futuros profesores de ciencias.

- Mejora de las concepciones de maestros en formación de la educación STEM.

- Reflexiones de futuro profesorado de física y química sobre naturaleza de la tecnología en el contexto de la controversia Tesla-Edison.

- Aprendizaje de microbiología experimental en un formato virtual en contexto de pandemia: una experiencia de implementación de actividades con la utilización de un set portátil en la formación de profesores de Biología y Ciencias Naturales.

La profesora Carmen Ropero-Padilla y sus colegas (Universidad Jaume I, España) presentan un estudio cualitativo en el que participan profesores de ciencias en activo con el objetivo de analizar sus estilos y estrategias de enseñanza.

- Aproximación a los estilos y estrategias de enseñanza del profesorado de la ESO y Bachillerato de ciencias experimentales: un estudio de caso.

También hallarán experiencias educativas en el marco de la educación científica que adoptan enfoques y/o recursos didácticos innovadores como las presentadas por el profesor Roberto Irineu da Silva et al. (Colégio Pedro II; Universidade Federal Fluminense, Brasil); y Jefferson Silva-Costa
(Universidade de Burgos, Espanha) e Diego Armando Retana (Universidade da Costa Rica, Costa Rica) centram-se no conhecimento didático dos futuros professores. Por outro lado, o professor Antonio García-Carmona (Universidade de Sevilha, Espanha) e a professora María José VargasStraube e os seus colaboradores (Universidade Alberto Hurtado, Chile) abordam o conhecimento científico e tecnológico dos futuros professores de Ciências.

- Aperfeiçoamento dos conceitos dos professores em formação das áreas STEM.

- Reflexões dos estudantes de Licenciatura em Física e Química sobre a natureza da tecnologia no contexto da batalha das correntes Tesla vs. Edison.

- Aprendizagem de microbiologia experimental em formato virtual no contexto de pandemia: uma experiência de implementação de atividades com o uso de um laboratório portátil na formação de professores de Biologia e Ciências Naturais.

A professora Carmen Ropero-Padilla e colegas (Universidade Jaume I, Espanha) apresentam um estudo qualitativo envolvendo professores de Ciências em exercício, com o objetivo de analisar os seus estilos e estratégias de ensino.

- Aproximação aos estilos e estratégias de ensino de professores da Educação Secundária Obrigatória (ESO) e do Ensino Médio (Bachillerato) em Ciências Experimentais: um estudo de caso.

Também há experiências educacionais no âmbito da educação científica que adotam abordagens e/ou recursos didáticos inovadores, como as apresentadas pelo professor Roberto Irineu da Silva et al. (Colégio Pedro II; Universidade Federal Fluminense, Brasil); e Jefferson Silva-Costa e Tereza Cristina Cavalcanti (Universidade Federal de Alagoas, Brasil). Todas elas estão intimamente relacionadas às Tecnologias da Informação e Comunicação (TIC). 
y Tereza Cristina Cavalcanti (Universidade Federal de Alagoas, Brasil). Todas ellas están estrechamente relacionadas con las Tecnologías de la Información y la Comunicación (TIC).

- Videoaulas acessíveis sobre a temática água como recurso didático-pedagógico para promoção da educação científica.

- Estamos sendo invadidos. Discutindo sobre os conceitos científicos relacionados à pandemia de COVID-19 através da elaboração de memes.

La profesora Lourdes Aragón y sus colaboradoras (Universidad de Cádiz, España) centran sus miras en la etapa de Educación Infantil, olvidada habitual en la Didáctica de las Ciencias Experimentales. Así, presentan un estudio empírico sobre el análisis del discurso científico en alumnos y docentes de Educación Infantil, el cual fue diseñado a partir de una experiencia educativa basada en el huerto escolar como recurso didáctico.

- Sostener el discurso científico en aulas de 4 y 5 años: análisis de las intervenciones de docentes y alumnado y del conocimiento emergente en torno al huerto ecológico escolar.

Por último, desde una perspectiva más teórica, encontrarán las aportaciones del profesor Anderson Moser y sus colegas (Universidade Federal do Paraná, Brasil) y del profesor Javier Arabit y su equipo (Universidad de Murcia, España). Ambas valoran las oportunidades que puede ofrecer la Tecnología Educativa en la educación científica.

- A emergência climática no ensino de Ciências: os saberes necessários para uma proposta de trabalho pedagógico por meio da educomunicação científica.

- Uso de tecnologías avanzadas para la educación científica.

Reseñados los artículos incorporados en este primer monográfico, nos encontramos con temáticas científicas relativas a la Biología y la Física, además de otras transversales
- Videoaulas acessíveis sobre a temática da água como recurso didático-pedagógico para promoção da educação científica.

- Estamos sendo invadidos. Discutindo sobre os conceitos científicos relacionados à pandemia de COVID-19 através da elaboração de memes.

A professora Lourdes Aragón e as suas colaboradoras (Universidade de Cádiz, Espanha) concentram a atenção na etapa da Educação Infantil, que normalmente é esquecida na Didática das Ciências Experimentais. Apresentam um estudo empírico sobre a análise do discurso científico de alunos e professores de Educação Infantil, elaborado a partir de uma experiência educacional baseada na horta escolar como um recurso didático.

- Sustentar o discurso científico em salas de aula de alunos de 4 e 5 anos: análise das intervenções de professores ealunos e dos conhecimentos emergentes sobre a horta da escola.

Finalmente, sob uma perspectiva mais teórica, apresentamos as contribuições do professorAnderson Moser e colegas (Universidade Federal do Paraná, Brasil) e do professor Javier Arabit e equipe (Universidade de Murcia, Espanha). Ambos destacam as oportunidades quea Tecnologia Educacional pode oferecer na educação científica.

- A emergência climática no ensino de Ciências: os saberes necessários para uma proposta de trabalho pedagógico por meio da educomunicação científica.

- Uso de tecnologias avançadas para a educação científica.

Os artigos incluídos neste primeiro volume abordam temas científicos relativos à Biologia e à Física, assim como outros temas transversais como Emergência Climática, o COVID, a Horta Escolar e a Água. O público-alvo das pesquisas e inovações abrange desde alunos de Educação Infantil 
como la Emergencia Climática, el COVID, el Huerto Ecológico y el Agua. Igualmente, los destinatarios de las investigaciones e innovaciones van desde escolares de Educación Infantil a docentes en formación o en activo. En cuanto a la naturaleza de los artículos ésta incluye, desde los enfoques cualitativos a los cuantitativos y mixtos. Encontrarán asimismo metodologías en torno al modelo aula invertida, la argumentación científica, la educomunicación o la educación inclusiva.

Confiamos en que, tanto este primer ejemplar del monográfico como el que se publicará más adelante, cumplan con los objetivos inicialmente planteados y sirvan de acicate para iniciar o consolidar propuestas de trabajo fundamentadas y novedosas, en el afán de implicar a los estudiantes y profesorado en el apasionante mundo del aprendizaje científico. até professores em formação ou em exercício. Quanto à natureza dos artigos, varia de abordagens qualitativas a quantitativas e mistas. Há também metodologias em torno do modelo de sala de aula invertida, argumentação científica, educomunicação e educação inclusiva.

Acreditamos que tanto este primeiro exemplar da edição monográfica quanto o que será publicado posteriormente atingirão os objetivos inicialmente estabelecidos e servirão de estímulo para iniciar ou consolidar propostas de trabalho bem fundamentadas e inovadoras, desejando implicar estudantes e professores no apaixonante mundo do aprendizado científico. 